\title{
Dark Matter Searches at ATLAS
}

\author{
Sascha Mehlhase \\ on behalf of the ATLAS Collaboration ${ }^{1, a)}$
}

${ }^{1}$ Ludwig-Maximilians-Universität München

a) sascha.mehlhase@physik.uni-muenchen.de

\begin{abstract}
The large excess of Dark Matter observed in the Universe and its particle nature is one of the key problems yet to be solved in particle physics. Despite the extensive success of the Standard Model, it is not able to explain this excess, which instead might be due to yet unknown particles, such as Weakly Interacting Massive Particles, that could be produced at the Large Hadron Collider. This contribution will give an overview of different approaches to finding evidence for Dark Matter with the ATLAS experiment in $\sqrt{s}=8 \mathrm{TeV}$ Run-1 data.
\end{abstract}

\section{Introduction}

While the existence of Dark Matter in the Universe is well established through numerous astrophysical and cosmological observations [1-4], little is known of its particle nature or its non-gravitational interactions. At the Large Hadron Collider, one can search for a weakly interacting massive particle (WIMP), denoted by $\chi$, and for interactions between $\chi$ and Standard Model particles [5]. Searches conducted at the Large Hadron Collider are especially sensitive at low Dark Matter masses $\left(m_{\chi} \leq 10 \mathrm{GeV}\right)$, and therefore provide results complementary to direct Dark Matter searches [6-9].

The interaction of particles mediating between Dark Matter and Standard Model particles can be described by contact operators in the framework of an effective field theory (EFT) [10-12] in cases where they are too heavy to be produced directly in the experiment. In the absence of signal, limits can be placed in terms of the effective mass scale of the interaction, $M_{*}$, and of the $\chi$-nucleon cross-section, $\sigma_{\chi-N}$, as a function of $m_{\chi}$. In addition to the investigation of EFT operators, pair production of WIMPs is also investigated within so-called simplified models, where a pair of WIMPs couples to a pair of Standard Model particles explicitly via a new mediator particle, e.g. a new vector boson $Z^{\prime}$. In this case, limits on $M_{*}$ and/or $m_{\chi}$ are placed as a function of the mediator mass $M_{\text {med }}$.

A number of dedicated approaches to finding evidence for Dark Matter have been carried out with the ATLAS experiment [13] during Run 1, using typically about $20 \mathrm{fb}^{-1}$ of data collected at a centre-of-mass energy of $\sqrt{s}=$ $8 \mathrm{TeV}$. So-called mono-X searches take advantage of a variety of different tag objects, $\mathrm{X}$, together with large absolute values of missing transverse momentum, $E_{\mathrm{T}}^{\mathrm{miss}}$, in the final state to constitute a clean and distinctive signature. Heavyquark searches use events with large $E_{\mathrm{T}}^{\mathrm{miss}}$ in association with high-momentum jets of which one or more are identified as jets containing $b$-quarks. Finally, Higgs-to-invisible searches look at invisible Higgs-boson decays, in events with large $E_{\mathrm{T}}^{\text {miss }}$ and either decay products of a vector boson produced in association with the Higgs boson, or remnant jets resulting from vector boson fusion (VBF) production of the Higgs boson.

\section{Mono-X Searches}

Tagging events using a variety of recoil objects, mostly stemming from initial state radiation (ISR), gives access to a broad range of EFT operators, as the respective sensitivity depends on the tag object in question. All cases require large amounts of $E_{\mathrm{T}}^{\text {miss }}$, stemming from the Dark Matter particle, and tag objects include single jets or photons as well as $Z$ and $W$ bosons. 

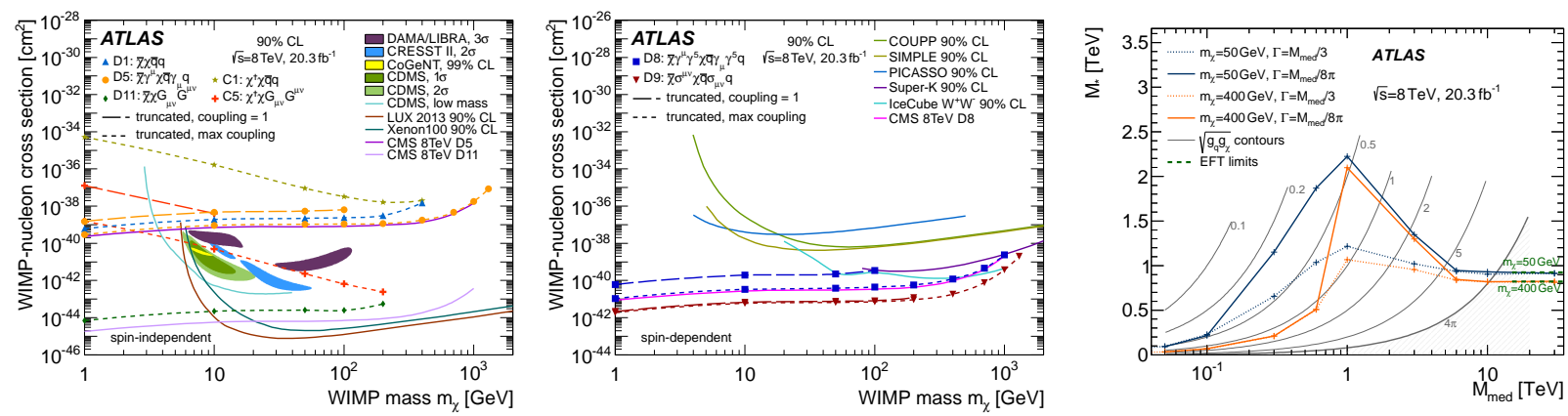

FIGURE 1. Inferred $90 \% \mathrm{CL}$ limits on (left) the spin-independent and (middle) spin-dependent $\sigma_{\chi-N}$ as a function of $m_{\chi}$ for different operators. Observed 95\% CL limits on $M_{*}$ as a function of $M_{\text {med }}$ (right), assuming a $Z^{\prime}$-like boson in a simplified model and a Dark Matter mass of $50 \mathrm{GeV}$ and $400 \mathrm{GeV}$. The width of the mediator is varied between $M_{\text {med }} / 3$ and $M_{\text {med }} / 8 \pi$. The corresponding limits from EFT models are shown as dashed lines; contour lines indicating a range of values of the product of the coupling constants $\left(\sqrt{g_{q} g_{\chi}}\right)$ are also shown. $[6,9,14-29]$

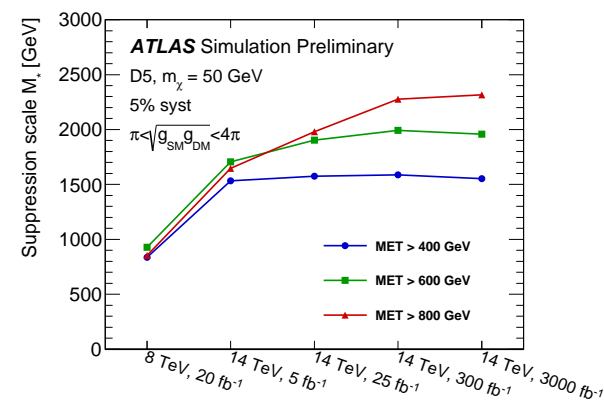

FIGURE 2. The 95\% CL lower limits on the suppression scale $M_{*}$ at $\sqrt{s}=8 \mathrm{TeV}$ and $14 \mathrm{TeV}$ for three signal regions defined by $E_{\mathrm{T}}^{\text {miss }}>400 \mathrm{GeV}, 600 \mathrm{GeV}$ and $800 \mathrm{GeV}$. The limits are shown for the D5 operators with $m_{\chi}=50 \mathrm{GeV}$ assuming $5 \%$ total background systematic uncertainty. The $8 \mathrm{TeV}$ limits presented are based on simulation only. These results assume that the EFT is a valid approach. [30]

\section{Mono-jet $+E_{\mathrm{T}}^{\text {miss }}$}

As introduced in [14], using a single jet as recoil object gives sensitivity to six EFT operators (D1, D5, D8, D9, D11 and C5). To enhance the expected signal, events are required to contain at least one central jet with transverse momentum, $p_{\mathrm{T}}$, larger than both $120 \mathrm{GeV}$ and half of $E_{\mathrm{T}}^{\text {miss }}$. To ensure that the jet is in fact recoiling against the Dark Matter particles, the angle between the jet and the missing transverse momentum in the events is required to be above one. To further suppress background, which is mainly comprised of $Z(v v)+$ jets and $W(\ell v)+$ jets events, events containing leptons or high- $p_{\mathrm{T}}$ isolated tracks are vetoed. As all measurements are consistent with Standard Model expectations, the most sensitive out of nine signal regions, defined by requirements on $E_{\mathrm{T}}^{\text {miss }}$ ranging from $150 \mathrm{GeV}$ to $700 \mathrm{GeV}$, is used to place limits on $\sigma_{\chi-N}$ for each of the operators under investigation. As an example result of this analysis, inferred $90 \%$ confidence level (CL) limits on $\sigma_{\chi-N}$ as a function of $m_{\chi}$ for the spin-independent as well as spin-dependent case for different operators are shown in Fig. 1 (left \& middle). To ensure the validity of the EFT approach, the results are also shown after applying a truncation procedure as described in [14]. Limits on $M_{*}$ as a function of $M_{\text {med }}$ in the context of a simplified model are shown in Fig. 1 (right).

Prospects for a mono-jet search in Run 2, yielding a significantly increased sensitivity especially due to higher centreof-mass energy, have been studied and presented in [30]. As an example, Fig. 2 illustrates the estimated reach for $M_{*}$ as a function of the centre-of-mass energy and integrated luminosity. 

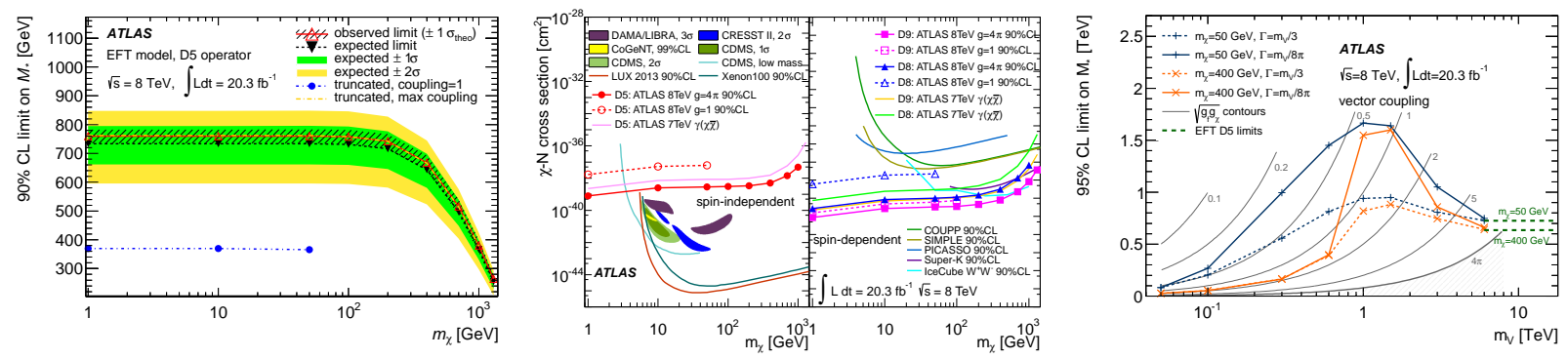

FIGURE 3. Limits at $90 \% \mathrm{CL}$ on $M_{*}$ as a function of $m_{\chi}$ (left), for the vector operator D5. Results where EFT truncation is applied (see text) are also shown, assuming coupling values $\sqrt{g_{f} g_{\chi}}=1,4 \pi$. Upper limits at $90 \%$ CL on $\sigma_{\chi-N}$ as a function of $m_{\chi}$ for spin-independent (middle-left) and spin-dependent (middle-right) interactions, for a coupling strength $\sqrt{g_{f} g_{\chi}}$ of unity or the maximum value $(4 \pi)$ that keeps the model within its perturbative regime. The truncation procedure is applied for both cases. Observed lower limits at $95 \% \mathrm{CL}$ on $M_{*}$ as a function of $M_{\text {med }}$ (right), for a $Z^{\prime}$-like mediator with vector interactions. For an $m_{\chi}$ of $50 \mathrm{GeV}$ or $400 \mathrm{GeV}$, results are shown for different values of the mediator total decay width $\Gamma$ and compared to the EFT observed limit results for a D5 (vector) interaction. $M_{*}$-vs- $m_{V}$ contours for an overall coupling $\sqrt{g_{f} g_{\chi}}=0.1,0.2,0.5,1,2,5,4 \pi$ are also shown. The corresponding limits from the D5 operator are shown as a dashed line. $[6,8,9,20,21,24,26,29,31-36]$

\section{Mono-photon $+E_{\mathrm{T}}^{\mathrm{miss}}$}

A search using a photon as tag object, able to access three EFT operators (D5, D8 and D9), has been shown in [31]. Using events with a single highly energetic photon, large $E_{\mathrm{T}}^{\text {miss }}$, no leptons and at most one jet; the main backgrounds in this analysis remain $Z(v v)+\gamma$, diboson $W \gamma$ and $Z \gamma$ with lost leptons as well as $W$ and $Z$ production with leptons misidentified as photons. As for the mono-jet search, all measurements are consistent with Standard Model expectations and lower (upper) limits on $M_{*}\left(\sigma_{\chi-N}\right)$ are presented both with and without applying the EFT truncation procedure mentioned above, as shown in examples in Fig. 3 (left and middle). In addition limits on $M_{*}$ as a function of $M_{\text {med }}$, as shown in Fig. 3 (right), are derived in the context of a simplified model.

\section{Mono- $W / Z+E_{\mathrm{T}}^{\text {miss }}$}

An analysis using $W$ and $Z$ bosons, respectively their decay products, as tag objects gives sensitivity to four EFT operators (C1, D1, D5 and D9) and has been presented in [37-39], for both hadronic and leptonic decays of the vector bosons. In the analysis aiming at hadronic decays, events are required to contain at least one high- $p_{\mathrm{T}}$ large-radius jet with reasonably balanced subjets, originating from the vector boson; at most one additional regular jet; and no leptons and photons. The background yield in the two signal regions, defined by requirements on $E_{\mathrm{T}}^{\text {miss }}$ of $350 \mathrm{GeV}$ and $500 \mathrm{GeV}$, are dominated by $Z(v v)+$ jets as well as $Z(\ell \ell)+$ jets and $W(\ell v)+$ jets with lost leptons. Looking at leptonic decays, the event selection differs for $W$ and $Z$ bosons. For the former, events are required to contain exactly one high$p_{\mathrm{T}}$ lepton and a transverse mass of the $W$ boson candidate incompatible with direct production; while in the latter case, events have to contain two leptons giving an invariant mass close to the $Z$ peak and no additional lepton or jets. Both cases require large values of $E_{\mathrm{T}}^{\text {miss }}$ and the main background contributions are coming from diboson events in the $Z$ case and in addition $W(\ell v)$ and $Z(\ell \ell)$ with lost leptons in the $W$ case. All yields are consistent with Standard Model expectations and limits on $\sigma_{\chi-N}$, as shown in Fig. 4, are presented both for spin-independent and spin-dependent EFT operators.

\section{Heavy-quarks Searches}

A search for Dark Matter pair production in association with bottom or top quarks has been presented in [40]. Aside from being sensitive to three EFT operators (C1, D1 and D9), this analysis also places constraints on the mass of a coloured mediator suitable to explain a possible signal of annihilating Dark Matter, using a simplified model approach. Several signal regions are defined requiring combinations of increasing jet and $b$-jet multiplicity, 0 or 1 lepton and values of $E_{\mathrm{T}}^{\text {miss }}$ above $200 \mathrm{GeV}$ to $300 \mathrm{GeV}$ in the events. Applying additional kinematic cuts, the main background is still coming from $t \bar{t}$-events as well as single-top and $W / Z+$ jets events. All measurements are consistent with Standard Model expectations and lower (upper) limits on $M_{*}\left(\sigma_{\chi-N}\right)$ are presented for three EFT operators (C1, D1 and D9), as 


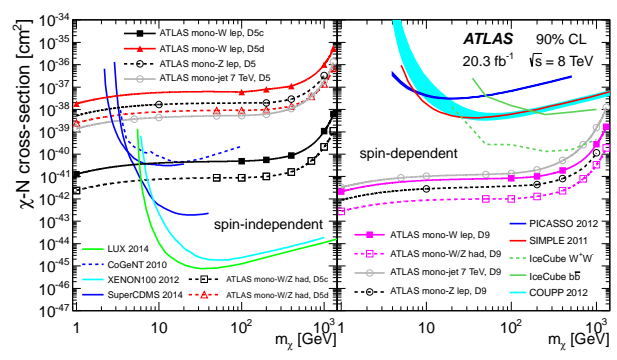

FIGURE 4. Observed limits on $\sigma_{\chi-N}$ as a function of $m_{\chi}$ at $90 \%$ CL for spin-independent (left) and spin-dependent (right) operators in the EFT. [6-9, 29, 30, 32, 34, 35, 37, 38]
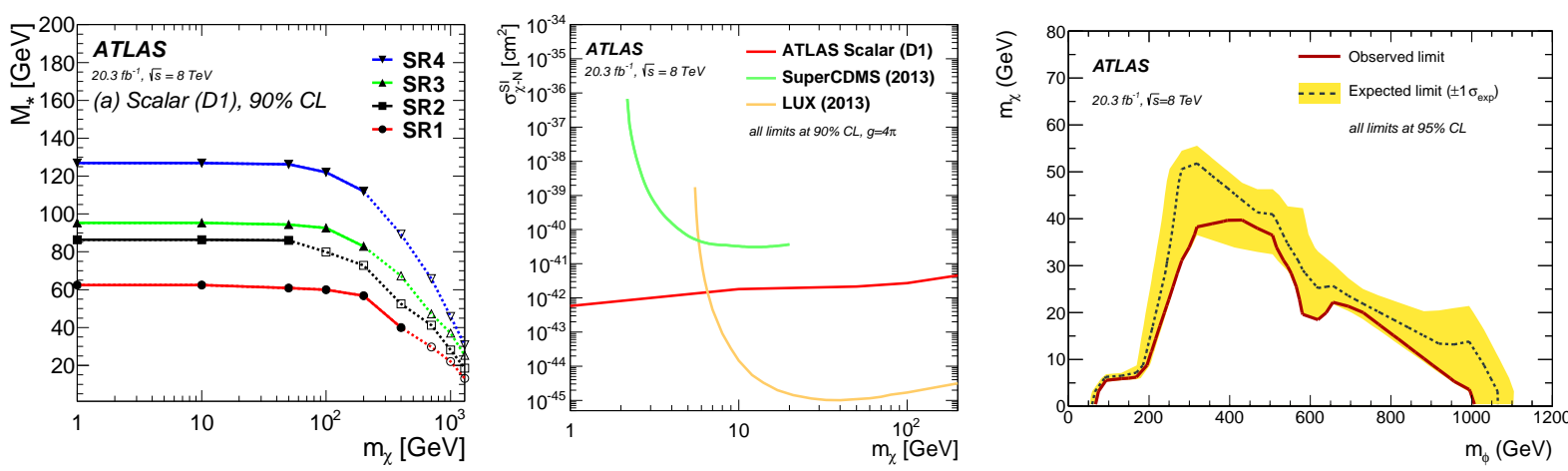

FIGURE 5. Lower limits on $M_{*}$ at $90 \% \mathrm{CL}$ for different signal regions as a function of $m_{\chi}$ (left) for the operator D1. Solid lines and markers indicate the validity range of the effective field theory assuming couplings $g_{q} g_{\chi}<4 \pi$, the dashed lines and hollow makers represent the full collider constraints. Upper limits at 90\% CL on $\sigma_{\chi-N}$ for the scalar operator D1 as a function of $m_{\chi}$ (middle) compared to other results. The coupling is assumed to be $g_{q} g_{\chi}=4 \pi$. Exclusion contour at $95 \%$ CL for the $b$-flavoured Dark Matter model (right) from combined results of two signal regions. The expected limit is given by the dashed line, and the yellow band indicates the $\pm 1 \sigma$ uncertainty. The observed limit is given by the solid red line. The region beneath the curve indicating the observed limit is excluded. $[6,7,40,43]$

show for D1 in Fig. 5 (left and middle). Due to the proportionality of the scalar operator to the quark mass, limits for D1 are in fact better than those obtained by the above mentioned mono-jet analysis. Constraints on $b$-flavoured Dark Matter models, using a simplified model, are also presented and shown in Fig. 5 (right). For a Dark Matter particle of about $35 \mathrm{GeV}$, as suggested by an interpretation [41] of data recorded by the Fermi-LAT collaboration [42], mediator masses between roughly $300 \mathrm{GeV}$ and $500 \mathrm{GeV}$ are excluded at $95 \% \mathrm{CL}$.

\section{Higgs-to-invisible Searches}

In a variety of extensions of the Standard Model, the recently discovered Higgs boson can invisibly decay to a pair of Dark Matter particles if kinematically allowed. Analyses aiming at vector bosons produced in association with an invisible Higgs boson [44, 45] as well as invisible Higgs bosons produced via VBF [46] have been presented. In the former case both leptonic and hadronic decays of $W$ bosons as well as leptonic decays of $Z$ bosons have been investigated and limits on the branching ratio (BR) of invisible Higgs boson decays have been placed at 78\% and $75 \%$, respectively. 95\% CL upper limits on the product of the $V H$ cross sections and the $V(j j)$ and $H$ (inv) decay branching ratio, $\sigma_{\mathrm{VH}} \times \mathrm{BR}(\mathrm{H} \rightarrow$ inv. $)$, as a function of the Higgs boson mass in the range $115 \mathrm{GeV}<m_{H}<300 \mathrm{GeV}$, are shown in Fig. 6 (left), while 95\% CL upper limits on $\sigma_{\mathrm{ZH}} \times \mathrm{BR}\left(\mathrm{H} \rightarrow\right.$ inv.) in the mass range $110 \mathrm{GeV}<m_{H}<400 \mathrm{GeV}$ are shown in Fig. 6 (middle). Under assumptions detailed in [45], the latter results can also be reinterpreted as limits on $\sigma_{\chi-N}$ as a function of $m_{\chi}$ (right) at $90 \% \mathrm{CL}$, extracted from the $\mathrm{BR}(\mathrm{H} \rightarrow$ inv.) limit in a Higgs-portal scenario, as shown in Fig. 6 (right). Using the approach via VBF production of an invisible Higgs boson, the upper limit on the branching ratio of invisible Higgs boson decays is set at 29\%, with $35 \%$ expected, as shown in [46]. 

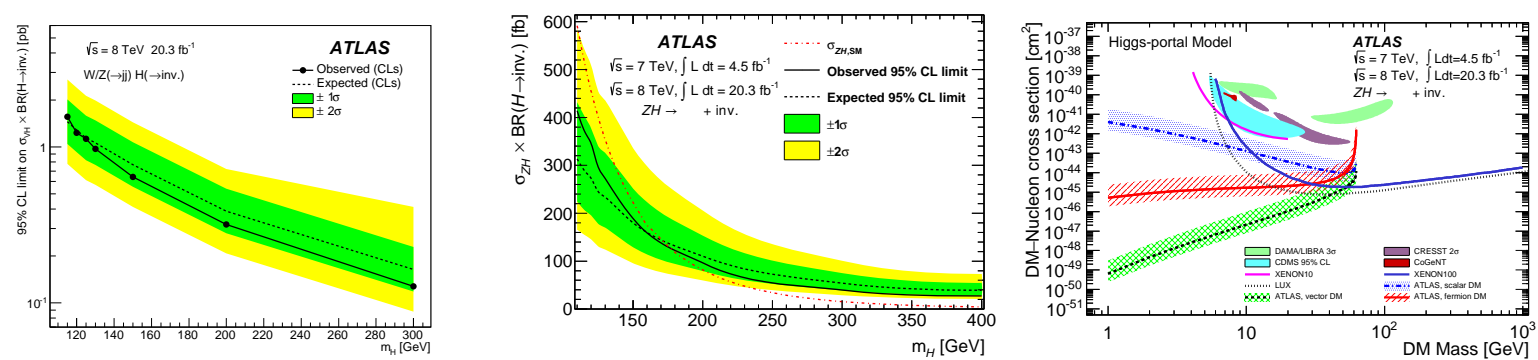

FIGURE 6. Upper limits on $\sigma_{\mathrm{VH}} \times \mathrm{BR}\left(\mathrm{H} \rightarrow\right.$ inv.) (left) at $95 \% \mathrm{CL}$ for a Higgs boson with $115 \mathrm{GeV}<m_{H}<300 \mathrm{GeV}$. The full and dashed lines show the observed and expected limits, respectively. Upper limits on $\sigma_{\mathrm{ZH}} \times \mathrm{BR}(\mathrm{H} \rightarrow$ inv.) (middle) at $95 \%$ CL for a Higgs boson with $110 \mathrm{GeV}<m_{H}<400 \mathrm{GeV}$, for the combined 7 and $8 \mathrm{TeV}$ data. The full and dashed lines show the observed and expected limits, respectively. Limits on $\sigma_{\chi-N}$ as a function of $m_{\chi}$ (right) at 90\% CL, extracted from the BR(H $\rightarrow$ inv.) limit in a Higgs-portal scenario, compared to other results. Cross-section limits and favoured regions correspond to a $90 \%$ CL, unless stated otherwise in the legend. [6, 20, 21, 24, 32, 44, 45, 47-49]

\section{Conclusion}

The ATLAS Collaboration has performed a broad variety of searches for Dark Matter signatures, using $\sqrt{s}=8 \mathrm{TeV}$ Run-1 data and tag objects ranging from single jets and photons, to $W / Z$ bosons and heavy quarks as well as looking for variations in the branching ratio of invisible Higgs boson decays. No signs of Dark Matter have been observed, and stringent limits have been set on different benchmark models, emphasising the complementary nature of collider searches to direct and indirect detection experiments especially at low Dark Matter masses and for spin-dependent EFT operators.

In Run 2, already started in mid 2015, the Large Hadron Collider provides proton collisions at a centre-of-mass energy of $\sqrt{s}=13 \mathrm{TeV}$, making a much higher energy scale accessible to these searches. Over the foreseen run time, ATLAS will collect three to four times the data collected in Run 1. Both, higher centre-of-mass energy and higher luminosity, will result in a significantly increased sensitivity and should enable ATLAS to discover signs of physics beyond the Standard Model or further improve all of the limits set above.

\section{REFERENCES}

[1] F. Zwicky, Helv. Phys. Acta 6, 110-127 (1933).

[2] G. Bertone et al., Phys. Rept. 405, 279-390 (2005), arXiv:hep-ph/0404175 [hep-ph] .

[3] G. Jungman et al., Phys. Rept. 267, 195-373 (1996), arXiv:hep-ph/9506380 [hep-ph] .

[4] J. Binney and S. Tremaine, (1993), arXiv:astro-ph/9304010 [astro-ph] .

[5] J. Beringer et al. (Particle Data Group), Phys. Rev. D 86, p. 010001Jul (2012).

[6] D. S. Akerib et al. (LUX), Phys. Rev. Lett. 112, p. 091303 (2014), arXiv:1310.8214 [astro-ph.CO] .

[7] R. Agnese et al. (SuperCDMS), Phys. Rev. Lett. 112, p. 041302 (2014), arXiv:1309.3259 [physics.ins-det] .

[8] E. Behnke et al. (COUPP Collaboration), Phys. Rev. D 86, p. 052001Sep (2012).

[9] S. Archambault et al. (PICASSO), Phys. Lett. B711, 153-161 (2012), arXiv:1202.1240 [hep-ex] .

[10] M. Beltran et al., JHEP 09, p. 037 (2010), arXiv:1002.4137 [hep-ph] .

[11] P. J. Fox et al., Phys. Rev. D85, p. 056011 (2012), arXiv:1109.4398 [hep-ph] .

[12] J. Goodman et al., Phys. Rev. D82, p. 116010 (2010), arXiv:1008.1783 [hep-ph] .

[13] ATLAS Collaboration, JINST 3, p. S08003 (2008).

[14] ATLAS Collaboration, Eur. Phys. J. C75, p. 299 (2015), arXiv:1502.01518 [hep-ex] .

[15] CMS Collaboration, Eur. Phys. J. C75, p. 235 (2015), arXiv:1408.3583 [hep-ex] .

[16] P. A. R. Ade et al. (Planck), Astron. Astrophys. 571, p. A16 (2014), arXiv:1303.5076 [astro-ph.CO] .

[17] G. Hinshaw et al., ApJS 208, p. 19 (2013), arXiv:1212.5226.

[18] M. Ackermann et al. (Fermi-LAT), Phys. Rev. D89, p. 042001 (2014), arXiv:1310.0828 [astro-ph.HE] .

[19] A. Abramowski et al. (H.E.S.S. Collaboration), Phys. Rev. Lett. 106, p. 161301Apr (2011).

[20] G. Angloher et al., Eur. Phys. J. C72, p. 1971 (2012), arXiv:1109.0702 [astro-ph.CO] . 
[21] R. Agnese et al. (CDMS), Phys. Rev. Lett. 111, p. 251301 (2013), arXiv:1304.4279 [hep-ex] .

[22] R. Agnese et al. (SuperCDMS), Phys. Rev. Lett. 112, p. 241302 (2014), arXiv:1402.7137 [hep-ex] .

[23] C. E. Aalseth et al., (2014), arXiv:1401.6234 [astro-ph.CO] .

[24] R. Bernabei et al. (DAMA), Eur. Phys. J. C56, 333-355 (2008), arXiv:0804.2741 [astro-ph] .

[25] E. Aprile et al. (XENON100), Phys. Rev. Lett. 111, p. 021301 (2013), arXiv:1301.6620 [astro-ph.CO] .

[26] S. Desai et al. (Super-Kamiokande), Phys. Rev. D70, p. 083523 (2004), arXiv:hep-ex/0404025 [hep-ex] .

[27] R. Abbasi et al. (IceCube Collaboration), Phys. Rev. Lett. 102, p. 201302May (2009).

[28] E. Behnke et al. (COUPP Collaboration), Phys. Rev. Lett. 106, p. 021303Jan (2011).

[29] M. Felizardo et al. (The SIMPLE Collaboration), Phys. Rev. Lett. 108, p. 201302May (2012).

[30] ATLAS Collaboration, ATL-PHYS-PUB-2014-007 (2014), https://cds.cern.ch/record/1708859 .

[31] ATLAS Collaboration, Phys. Rev. D91, p. 012008 (2015), arXiv:1411.1559 [hep-ex] .

[32] E. Aprile et al. (XENON100 Collaboration), Phys. Rev. Lett. 109, p. 181301Nov (2012).

[33] Z. Ahmed et al. (CDMS Collaboration), Phys. Rev. Lett. 106, p. 131302Mar (2011).

[34] C. E. Aalseth et al. (CoGeNT Collaboration), Phys. Rev. Lett. 106, p. 131301Mar (2011).

[35] M. G. Aartsen et al. (IceCube Collaboration), Phys. Rev. Lett. 110, p. 131302Mar (2013).

[36] A. Abramowski et al. (HESS), Phys. Rev. Lett. 110, p. 041301 (2013), arXiv:1301.1173 [astro-ph.HE] .

[37] ATLAS Collaboration, Phys. Rev. Lett. 112, p. 041802 (2014), arXiv:1309.4017 [hep-ex] .

[38] ATLAS Collaboration, JHEP 09, p. 037 (2014), arXiv:1407.7494 [hep-ex] .

[39] ATLAS Collaboration, Phys. Rev. D90, p. 012004 (2014), arXiv:1404.0051 [hep-ex] .

[40] ATLAS Collaboration, Eur. Phys. J. C75, p. 92 (2015), arXiv:1410.4031 [hep-ex] .

[41] T. Daylan et al., (2014), arXiv:1402.6703 [astro-ph.HE] .

[42] W. B. Atwood et al., The Astrophysical Journal 697, p. 1071 (2009).

[43] R. Gaitskell et al., http://dmtools.brown.edu/ .

[44] ATLAS Collaboration, Eur. Phys. J. C75, p. 337 (2015), arXiv:1504.04324 [hep-ex] .

[45] ATLAS Collaboration, Phys. Rev. Lett. 112, p. 201802 (2014), arXiv:1402.3244 [hep-ex] .

[46] ATLAS Collaboration, ATLAS-CONF-2015-004 (2015), http://cds.cern.ch/record/2002121 .

[47] J. Angle et al. (XENON10 Collaboration), Phys. Rev. Lett. 107, p. 051301Jul (2011).

[48] C. E. Aalseth et al. (CoGeNT Collaboration), Phys. Rev. Lett. 107, p. 141301Sep (2011).

[49] P. J. Fox et al., Phys. Rev. D85, p. 036008 (2012), arXiv:1107.0717 [hep-ph] . 